\title{
Intensivmedizin up2date
}

$2 \cdot 2020$

\section{Allgemeine Intensivmedizin 1}

\section{Arzneimittelinteraktionen in der Intensivmedizin}




\section{Unter dieser Rubrik sind bereits erschienen:}

Delir bei Intensivpatienten S. Gurlit, C. Hempel,

C. Olotu-Steffen Heft $1 / 2020$

Hämodynamisches Monitoring in der Intensivmedizin G. Meyer, H. Ebelt Heft 1/2020

Nosokomiale Infektionen in der Intensivmedizin K. Schröppel Heft $1 / 2020$

Der schwierige Atemweg auf der Intensivstation

T. Kandaouroff, H.-G. Bone Heft 1/2020

Intensivmedizinisches Infektions- und Ausbruchsmanagement

M. Herz, D. Pape, A. Friedrichs Heft 4/2019

Verhalten nach Komplikationen E. Biermann Heft 4/2019

Beatmungsstrategien bei chronisch obstruktiver Lungenerkrankung A. Kersten, M. Dreher Heft 3/2019

Notfall Sepsis: Erkennen, Diagnose, Therapie M. Gründling, S. Gibb, A. Kühn Heft 3/2019

Behandlung von Tuberkulose auf der Intensivstation J. Geiseler, K. Avsar Heft 3/2019

Akutes Leberversagen M. Busch, K. Stahl, M. P. Manns

Heft 2/2019

Volumentherapie C. S. Bruells, A. Schindler Heft 2/2019

Akutes Lungenversagen (ARDS) C. Lotz, P. M. Lepper,

R. M. Muellenbach Heft 2/2019

Point-of-Care-Sonografie in der Intensivmedizin C.-A. Greim Heft 2/2019

Beatmungsstrategien in der Intensivmedizin P. Spieth, C. Uhlig Heft $1 / 2019$

Neue Definitionen der Sepsis T. Schmoch, M. Bernhard, F. Uhle, J. Bickenbach, G. Marx, T. Brenner, M. A. Weigand Heft 4/2018
Fieber in der Intensivmedizin R. Kopp Heft 4/2018

Medizinisches Qualitätsmanagement: Qualitätsindikatoren O. Kumpf, R. Riessen, R. Dubb, A. Kaltwasser Heft 3/2018

Lungenersatzverfahren J. Bickenbach Heft 2/2018

Infektionen unter medikamentöser Immunsuppression bei transplantierten Intensivpatienten P. Lutz, C. P. Strassburg Heft 2/2018

Differenzierter Einsatz kardiovaskulär wirksamer Substanzen

S. Rehberg Heft 2/2018

Update: Immunsuppression bei Organtransplantationen D. Kniepeiss, A. R. Rosenkranz, P. Fickert, P. Schemmer Heft 1/2018

Diagnostik der Sepsis T. Schürholz Heft 1/2018

Der mangelernährte Patient auf der Intensivstation H.-G. Bone, C. Sandfeld, F. Kleynmans Heft 1/2018

Strukturierte Patientenübergabe D. J. Höchter, V. von Dossow Heft $1 / 2018$

Virale Atemwegserkrankungen - neue Viren M. Kleines Heft 4/2017

Innerklinisches Notfallmanagement J.-C. Schewe, S. Lenkeit, S. Seewald, B. Jakisch, T. Jantzen Heft 4/2017

High-Flow-Sauerstofftherapie in der Intensivmedizin M. Simon, J. Grensemann, S. Kluge Heft 3/2017

Prävention von Clostridium-difficile-Infektionen S. KolbeBusch Heft 3/2017

Pharmakokinetik bei Anwendung von Nierenersatzverfahren auf Intensivstation E. Michael, T. Dimski, D. Kindgen-Milles Heft 2/2017

\section{ALLES ONLINE LESEN}

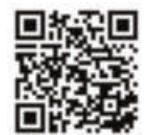

Mit der eRef lesen Sie Ihre Zeitschrift: online wie offline, am PC und mobil,

alle bereits erschienenen Artikel.

Für Abonnenten kostenlos! https://eref.thieme.de/intensiv-u2d

\section{IHR ONLINE-SAMMELORDNER}

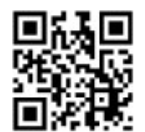

Sie möchten jederzeit und überall auf $\mathrm{Ihr}$ up2date-Archiv zugreifen? Kein Problem!

Ihren immer aktuellen Online-

Sammelordner finden Sie unter:

https://eref.thieme.de/EU18X

\section{JETZT FREISCHALTEN}

口:4i回 Sie haben Ihre Zeitschrift noch nicht freigeschaltet? Ein Klick genügt:

www.thieme.de/eref-registrierung 


\title{
Arzneimittelinteraktionen in der Intensivmedizin
}

Andreas Drust

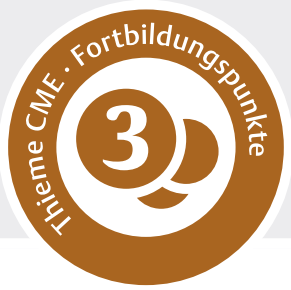

\begin{abstract}
Arzneimittelinteraktionen sind auf Intensivstationen ein häufiges Problem. Dabei spielt die Gesamtmenge der verschriebenen Medikamente bei kritisch kranken Patienten eine große Rolle: Durchschnittlich werden hier 25-35 Medikamente pro Patient eingesetzt. Fundierte Kenntnisse über entsprechende pharmakodynamische und pharmakokinetische Mechanismen sind für die Behandlungssicherheit daher essenziell.
\end{abstract}

\section{Definition und Häufigkeit}

Arzneimittelinteraktionen sind Wechselwirkungen zwischen 2 oder mehr Medikamenten mit Veränderung eines Wirkeffekts [1]. Diese können erwünscht sein, z. B. bei Arzneimittelsynergismen wie in der Helicobacterpylori-Eradikation oder der HIV-Therapie. Häufig sind Arzneimittelinteraktionen jedoch unerwünscht. Dabei ist ihre Auftretenswahrscheinlichkeit abhängig von der Anzahl der verordneten Medikamente [2]. Insbesondere alte Patienten sind regelmäßig von Polypharmakotherapie betroffen. Daten zeigen, dass 65-Jährige im Mittel 5 Medikamente gleichzeitig einnehmen [3]. In der Gruppe der 75- bis 84-Jährigen können es 6 und mehr sein [4].

Während des Aufenthalts auf einer Intensivstation erhalten Patienten durchschnittlich sogar 25-35 Arzneimittel [2]. Es ist daher verständlich, dass insbesondere in diesem klinischen Umfeld mit einem hohen Interaktionsrisiko gerechnet werden muss. Laut einer aktuellen Metaanalyse lässt sich bei $58 \%$ aller Intensivpatienten mindestens eine potenzielle Arzneimittelinteraktion detektieren, wobei diese bei jedem 6. betroffenen Patienten relevant verläuft. Umgekehrt lässt sich zeigen, dass je nach Einzeluntersuchung 7-44\% der klinisch relevanten unerwünschten Arzneimittelwirkungen interaktionsbedingt sind [5]. Kenntnisse wichtiger Wechselwirkungen sind für den Intensivmediziner daher für die Therapiesicherheit von großer Bedeutung.

\section{Einteilung}

Arzneimittelinteraktionen lassen sich in pharmakokinetische und pharmakodynamische Wechselwirkungen unterteilen. Die ebenfalls mit durchaus relevanter Häufigkeit auftretenden physikochemischen Inkompatibilitäten sollen in dieser Übersicht nicht beleuchtet werden. Es

\section{FALLBEISPIEL}

Teil 1: Patientenvorstellung und Anamnese

Eine 61-jährige Patientin $(157 \mathrm{~cm} ; 90 \mathrm{~kg})$ wird aufgrund einer therapieresistenten hypertensiven Krise von der gefäßchirurgischen Normalstation auf die Intermediate-Care-Station (IMC) übernommen. Einen Monat zuvor war eine aneurysmatisch erweiterte Anastomose der A. femoralis communis bei vorbestehender Bifurkationsprothese reseziert und durch ein iliako-(protheto-)profundales sowie femoro-(protheto-)femorales Interponat versorgt worden. In diesem Zug hatte sich eine Protheseninfektion entwickelt, weshalb die Patientin erneut stationär aufgenommen worden war. Nach erneuter Revisionsoperation mit Anlage eines Vakuumverbands hatten die Kollegen eine kalkulierte antiinfektive Therapie mit einer Kombination aus Meropenem, Vancomycin und Rifampicin begonnen.

wird zu deren weiterem Studium auf die einschlägige Literatur verwiesen.

\section{Pharmakokinetik}

Pharmakokinetische Interaktionen bedeuten Wechselwirkungen auf der Ebene des Weges des Arzneimittels durch den Körper. Dabei können Absorption, Verteilung, Metabolismus und Exkretion beeinflusst werden. Die größte klinische Bedeutung besitzen dabei Transport- und Metabolisierungsprozesse.

Das Transportprotein mit der wichtigsten klinischen Interaktionsrelevanz ist das MDR-1 (Multidrug-Resistance-Protein 1) oder auch p-Glykoprotein. Es wird vor- 
- Tab. 1 Substrate, Induktoren und Inhibitoren von p-Glykoprotein mit intensivmedizinischer Relevanz.

\begin{tabular}{l|l|l|}
\hline $\begin{array}{l}\text { p-Glykoprotein- } \\
\text { Substrate }\end{array}$ & $\begin{array}{l}\text { p-Glykoprotein- } \\
\text { Induktoren }\end{array}$ & $\begin{array}{l}\text { p-Glykoprotein- } \\
\text { Inhibitoren }\end{array}$ \\
\hline - Carvedilol & - Dexamethason & - Amiodaron \\
\hline - Digitoxin & - Phenytoin & - Itraconazol \\
\hline - Diltiazem, Verapamil & - Rifampicin & - Diltiazem, \\
- Dexamethason & & Verapamil \\
\hline - Ciclosporin, Siroli- & & - Ciclosporin, \\
mus, Tacrolimus & & Tacrolimus \\
\hline - Esomeprazol, Ome- & & - Clarithromycin, \\
prazol, Pantoprazol & & Erythromycin \\
\hline - Furosemid & \\
\hline - Midazolam & \\
\hline - Morphin & & \\
\hline - Phenytoin & & \\
\hline - Rifampicin & & \\
\hline
\end{tabular}

rangig in Leber, Niere, Darm, Gehirn, Plazenta und Hoden exprimiert. Die physiologische Funktion besteht dabei primär in einem zellulären Auswärtstransport xenogener Substanzen im Sinne einer Detoxifizierung [6]. Eine pharmakotherapeutische Hemmung von p-Glykoprotein führt in der Regel zu einer Erhöhung der Bioverfügbarkeit entsprechender Substrate, eine Induktion zu einer Verringerung. In Tab. 1 sind typische Substrate von p-Glykoprotein aufgeführt sowie Inhibitoren und Induktoren mit intensivmedizinischer Relevanz [7,8].

Weitere Transporter mit allgemeiner Relevanz für Arzneimittelinteraktionen sind das BRCP (Breast-Cancer-Resistance-Protein) sowie organische Anionen- und Kationentransporter. Für Wechselwirkungen bei kritisch Kranken spielen diese Proteine jedoch keine wesentliche Rolle [6].

Die Metabolisierung von Arzneimitteln lässt sich in 2 Phasen unterteilen:
In Phase I werden lipophile Substrate durch Oxidation, Reduktion oder Hydrolyse hydrophil, um anschließend in Phase II durch Konjugationsreaktionen deren renale Ausscheidungsfähigkeit zu erhöhen. Die Phase-I-Reaktionen erfolgen dabei durch Cytochrom-P450-abhängige Monooxygenasen (Cytochrom P450 = CYP). Von diesen existieren zahlreiche Subtypen, von denen insbesondere CYP3A4, CYP2C9, CYP2C19, CYP2D6 sowie CYP1A2 für den klinischen Alltag hinsichtlich eines Interaktionspotenzials besonders relevant sind. Das Cytochrom mit dem breitesten Biotransformationsspektrum ist CYP3A4. Etwa $50 \%$ aller Arzneistoffe werden durch dieses Protein metabolisiert $[9,10]$. In $~$ Tab. 2 sind typische Substrate von Cytochromen aufgelistet sowie Inhibitoren und Induktoren mit intensivmedizinischer Relevanz $[8,10]$.

Phase-II-Reaktionen spielen bei Interaktionen in der Intensivmedizin nur eine untergeordnete Rolle.

\section{Pharmakodynamik}

Neben pharmakokinetischen Interaktionen können auch Wechselwirkungen auf der Ebene der Pharmakodynamik auftreten. Diese lassen sich jedoch aufgrund ihres substanzspezifischen Wirkcharakters nicht in eine einfache Systematik einteilen [11]. Eine auf Intensivstationen typischerweise anzutreffende pharmakodynamische Interaktion ist eine Verlängerung der frequenzkorrigierten QTZeit (QTc) bei kombinierter Anwendung von Arzneimitteln mit entsprechendem Risikoprofil. Wichtig ist dabei jedoch, dass eine QTc-Zeit-Verlängerung allein nicht zwangsläufig zu einer klinischen Manifestation wie Torsade-de-Pointes führen muss. Umgekehrt sollte jedoch jede ventrikuläre Tachykardie im Sinne von Torsade-de-Pointes immer an eine mögliche Arzneimittelinteraktion denken lassen. - Tab. 3 zeigt typische QTc-Zeit-verlängernde Arzneimittel mit intensivmedizinischer Relevanz [12].

- Tab. 2 Substrate, Induktoren und Inhibitoren von Cytochrom P450 mit intensivmedizinischer Relevanz.

\begin{tabular}{|c|c|c|c|}
\hline Enzym & Substrate & Induktoren & Inhibitoren \\
\hline CYP1A2 & Haloperidol & Rifampicin, Carbamazepin & Ciprofloxacin \\
\hline CYP2C9 & Ibuprofen, Diclofenac, Naproxen & $\begin{array}{l}\text { Rifampicin, Phenytoin, } \\
\text { Carbamazepin }\end{array}$ & $\begin{array}{l}\text { Amiodaron, Metronidazol, } \\
\text { Fluconazol, Voriconazol }\end{array}$ \\
\hline CYP2C19 & Esomeprazol, Omeprazol & $\begin{array}{l}\text { Rifampicin, Phenytoin, } \\
\text { Carbamazepin }\end{array}$ & $\begin{array}{l}\text { Fluconazol, Voriconazol, } \\
\text { Esomeprazol, Omeprazol }\end{array}$ \\
\hline CYP2D6 & Haloperidol, Carvedilol, Metoprolol & nicht induzierbar! & Amiodaron \\
\hline CYP3A4 & $\begin{array}{l}\text { Fentanyl, Sufentanil, Buprenorphin, Hydromor- } \\
\text { phon, Morphin, Haloperidol, Midazolam, Dia- } \\
\text { zepam, Atorvastatin, Simvastatin, Methylpredni- } \\
\text { solon, Hydrocortison, Ciclosporin, Tacrolimus }\end{array}$ & $\begin{array}{l}\text { Rifampicin, Phenytoin, } \\
\text { Carbamazepin }\end{array}$ & $\begin{array}{l}\text { Clarithromycin, Erythromycin, Flu- } \\
\text { conazol, Itraconazol, Voriconazol, } \\
\text { Posaconazol, Diltiazem, Verapamil, } \\
\text { Amlodipin, Amiodaron }\end{array}$ \\
\hline
\end{tabular}


Eine seltene Komplikation in der Intensivmedizin ist das Serotoninsyndrom, welches u.a. als pharmakodynamische Interaktion auftreten kann. > Tab. 4 führt typische Arzneimittel auf, die mit einem Serotoninsyndrom assoziiert sind [13].

\section{Arzneimittel}

\section{Opioide}

Opioide sind Standardtherapeutika in der Intensivmedizin. Ihre Wirkungen entfalten sie über (partial-)agonistische Interaktionen mit $\mu$-, $\mathrm{k}$ - sowie $\delta$-Rezeptoren auf spinaler und supraspinaler Ebene [14]. Zur Analgosedierung werden insbesondere Fentanyl und Sufentanil eingesetzt. Zur Schmerztherapie finden v. a. Morphin, Piritramid, Hydromorphon und Buprenorphin Verwendung [15].

Fentanyl, Sufentanil und Buprenorphin sind Substrate von CYP3A4 und können daher durch relevante CYP3A4Inhibitoren wie Makrolidantibiotika, Azolantimykotika, Amiodaron oder Kalziumkanalblocker in ihrem Abbau gehemmt werden, mit entsprechend erhöhten Plasmaspiegeln. CYP3A4-Induktoren wie Rifampicin, Phenytoin oder Carbamazepin vermindern durch gesteigerte Biotransformation die Opioid-Plasmakonzentrationen [16,17].

Morphin und Hydromorphon sind Substrate der Uridindiphosphatglukuronyltransferase (UGT) und können durch deren Hemmung bzw. Induktion beeinflusst werden [18]. Diese potenziellen Interaktionen führen jedoch in der Regel zu wenig klinischen Problemen, da unzureichende Wirkungen bzw. Überdosierungen durch standardmäßiges Monitoring von Analgesie und Sedierung auf Intensivstationen schnell erkannt und die Opioiddosierungen entsprechend angepasst werden können [15].

Merke

Wechselwirkungen mit Einfluss auf die Plasmaspiegel von Opioiden und Sedativa können durch standardmäßiges Monitoring von Analgesie und Sedierung auf Intensivstationen schnell erkannt werden.

Es existieren Daten für Fentanyl und in geringem Umfang für Buprenorphin, die das Risiko eines Serotoninsyndroms belegen, wenn eine Kombination mit serotonergen Substanzen erfolgt [13]. In der Intensivmedizin kommen dafür v.a. antidepressiv wirkende Substanzen wie Trizyklika, selektive Serotoninwiederaufnahmehemmer (SSRI), Serotonin-Noradrenalin-Wiederaufnahmehemmer (SNRI) oder Mirtazapin in Frage - sei es aufgrund einer vorbestehenden antidepressiven Therapie oder im Rahmen einer Koanalgesie [19]. In diesem Zusammenhang sei auch darauf hingewiesen, dass das Oxazolidinon-Antibiotikum Linezolid eine klinisch relevante Monoaminoxidase hemmende Wirkung besitzt und daher
- Tab. 3 QTc-Zeit-verlängernde Arzneimittel mit intensivmedizinischer Relevanz.

\begin{tabular}{|l|l|l|}
\hline bekanntes Risiko & mögliches Risiko & bedingtes Risiko \\
\hline - Amiodaron & - Buprenorphin, & - Amantadin \\
\hline - Ciprofloxacin, Levo- & Hydrocodon & - Amphotericin B \\
\hline floxacin, Moxifloxacin & - Dexmede- & - Esomeprazol, Ome- \\
\hline - Clarithromycin, & tomidin & prazol, Pantoprazol \\
\hline Erythromycin & - Melperon & - Furosemid, \\
\hline - Domperidon & - Mirtazapin & Torasemid \\
\hline - Droperidol, & - Risperidon & - Hydrochlorothiazid \\
Haloperidol & - Tacrolimus & - Metoclopramid \\
\hline - Fluconazol & - Telavancin & - Metronidazol \\
\hline - Ondansetron & & - Piperacillin/ \\
\hline - Propofol & & Tazobactam \\
\hline - Sevofluran & & - Posaconazol, \\
\hline - Terlipressin & & Voriconazol \\
\hline
\end{tabular}

- Tab. 4 Intensivmedizinisch relevante Arzneimittel, die mit einem Serotoninsyndrom assoziiert sind.

\begin{tabular}{|l|l|}
\hline serotonerger Mechanismus & Arzneistoffe \\
\hline verminderter Serotoninabbau & - Linezolid \\
\hline Serotoninwiederaufnahme- & " selektive Serotoninwiederaufnahme- \\
hemmung & hemmer \\
& - Serotonin-Noradrenalin-Wiederauf- \\
& nahmehemmer \\
& - trizyklische Antidepressiva \\
& - Buprenorphin \\
& - Valproat \\
\hline & - Carbamazepin \\
& - Ondansetron \\
\hline Serotoninagonist & - Metoclopramid \\
\hline
\end{tabular}

ebenfalls in Kombination mit Fentanyl zu einem Serotoninsyndrom führen kann [20].

\section{Sedativa}

Propofol

Propofol ist neben Midazolam das am häufigsten eingesetzte Sedativum im Intensivbereich [15]. Es wird vermutet, dass seine hypnotische Potenz über eine Modulation der hemmenden Wirkung des Neurotransmitters Gammaaminobuttersäure (GABA) mittels ligandengesteuerter $G_{A B A}$-Rezeptoren hervorgerufen wird [21]. Propofol wird extensiv über diverse UGT und Cytochrome metabolisiert. Am besten untersucht ist CYP2B6. Patienten mit einer Variante dieses Enzyms (CYP2B6*6-Allel) besitzen eine reduzierte Biotransformationskapazität für Propofol mit entsprechender Kumulation [22]. Weiterhin können über eine UGT-Hemmung Propofol-Plasmaspiegel durch Valproat erhöht werden [23]. Zusätzlich sind pharmakodynamische Wechselwirkungen mit anderen 
sedierend wirkenden Arzneistoffen wie Clonidin, Dexmedetomidin, Opioiden oder Neuroleptika zu erwarten [15]. Propofol kann darüber hinaus allein oder in Komedikation mit weiteren Substanzen in seltenen Fällen eine Rhabdomyolyse auslösen. Insbesondere Kombinationen mit Statinen sowie Levofloxacin, Cotrimoxazol, Theophyllin, Risperidon, Venlafaxin, Mirtazapin und Gabapentin gelten dabei als Risikofaktoren [24].

Merke

Propofol zählt zu den Arzneimitteln mit nachgewiesener QTc-Zeit-verlängernder Wirkung [12]. Daher empfiehlt es sich, bei gemeinsamer Anwendung mit anderen QTc-Zeit-beeinflussenden Medikamenten ( $\bullet$ Tab. 3) auf EKG-Veränderungen besonders zu achten.

\section{Benzodiazepine}

Benzodiazepine entfalten ihre pharmakologischen Wirkungen über eine Modulation der hemmenden Wirkung des Neurotransmitters GABA mittels ligandengesteuerter $\mathrm{GABA}_{A}$-Rezeptoren [25]. Midazolam als intensivmedizinisch wichtigstes Benzodiazepin wird hauptsächlich über CYP3A4 metabolisiert. Sämtliche CYP3A4-Inhibitoren können daher prinzipiell zu einer Erhöhung der Midazolamspiegel führen. Klinische Daten diesbezüglich existieren insbesondere für Makrolidantibiotika, Azolantimykotika und Kalziumkanalblocker [8]. Im Gegensatz dazu führen Rifampicin und Phenytoin zu signifikant verminderten Midazolamspiegeln [10]. Wie auch bei Propofol sind pharmakodynamische Wechselwirkungen zwischen Midazolam und anderen sedierend wirkenden Arzneistoffen wie Clonidin, Dexmedetomidin, Opioiden oder Neuroleptika beschrieben [15].

Als weiteres Benzodiazepin spielt Diazepam intensivmedizinisch gelegentlich noch beim Alkoholentzugsdelir oder dem akuten Krampfanfall eine Rolle. Diazepam wird neben CYP3A4 auch in relevanten Mengen durch CYP2C19 metabolisiert [25]. Daher sind prinzipiell die gleichen pharmakokinetischen Interaktionen zu erwarten wie bei Midazolam. Darüber hinaus können durch die zusätzliche CYP2C19-Beteiligung starke Hemmstoffe dieses Enzyms wie Esomeprazol und Omeprazol zu einem signifikanten Anstieg des Diazepamspiegels führen. Für Esomeprazol wurden ein mehr als $80 \%$ iger Anstieg der Diazepam-AUC (Area Under the Curve) sowie eine Verdopplung der Eliminationshalbwertszeit beschrieben [8]. Sollte daher aus intensivmedizinischer Sicht ein Protonenhemmer gewünscht sein, empfiehlt sich in solchen Fällen am ehesten Pantoprazol, da dieses CYP2C19 nur unwesentlich beeinflusst [26].

Im Gegensatz zu Midazolam und Diazepam wird das noch häufig eingesetzte Lorazepam unwesentlich über das Cytochrom-P450-System metabolisiert. Die Biotransformation erfolgt allerdings in relevantem Ausmaß mittels verschiedener UGT [25]. UGT-Inhibitoren wie Fluconazol,
Ketamin, Morphin und Valproat zeigen in In-vitro-Untersuchungen allerdings keinen relevanten Einfluss auf den Lorazepam-Metabolismus [27]. Die häufig in Interaktionsdatenbanken beschriebene Abbauhemmung von Lorazepam durch Valproat mit der klinischen Empfehlung einer $50 \%$ igen Lorazepam-Dosisreduktion lässt sich laut einer aktuellen Übersichtsarbeit jedoch nicht ableiten [28]. Insgesamt kann Lorazepam im Intensivbereich als wenig interaktionsanfällig eingestuft werden.

\section{Dexmedetomidin}

Dexmedetomidin ist ein hochselektiver $\alpha_{2}$-Agonist mit zentral sedierender Komponente [29]. Es wird über CYP2A6, CYP1A2, CYP2E1, CYP2D6 und CYP2C19 metabolisiert und induziert diese auch. Die klinische Relevanz ist allerdings bislang unklar. Weiterhin hemmt es CYP2B6 und könnte daher theoretisch die Propofol-Clearance vermindern [30]. Bislang wurde dies jedoch nicht in entsprechenden Studien untersucht. Aus pharmakodynamischer Sicht muss bei kombinierter Anwendung mit einer verstärkten Wirkung weiterer sedierender Substanzen wie Opioiden, Propofol, Benzodiazepinen sowie volatilen Anästhetika gerechnet werden. Der typische bradykardisierende Effekt der $\alpha_{2}$-Agonisten wird durch Betablocker potenziert [29].

\section{Antibiotika}

Betalaktame

Betalaktamantibiotika hemmen die bakterielle Zellwandbiosynthese durch Blockade der Peptidoglykan-Quervernetzung und wirken daher bakterizid [31]. Die in der Intensivmedizin sehr häufig zur Anwendung kommenden Betalaktame werden oft bewusst mit weiteren antiinfektiven Substanzen kombiniert. Dies können Antibiotika anderer Substanzklassen oder Betalaktamaseinhibitoren sein. Ziel ist dabei stets eine Erweiterung des antibakteriellen Wirkungsspektrums. Es handelt sich daher um erwünschte pharmakodynamische Interaktionen. Für relevante unerwünschte Wechselwirkungen gelten Betalaktamantibiotika als eher weniger anfällig. Aus pharmakokinetischer Sicht interessant ist jedoch der beschleunigte Abbau von Aminoglykosiden unter Piperacillin-Komedikation. Ursächlich dafür sind offenbar direkte chemische Interaktionen zwischen Betalaktamring und Aminogruppen [32]. Da ein therapeutisches Drug-Monitoring von Aminoglykosiden heute weit verbreiteter Standard ist, sollte diese Interaktion klinisch allerdings kaum Probleme bereiten. Weiterhin ließ sich wiederholt zeigen, dass Carbapeneme die Plasmaspiegel von Valproat deutlich reduzieren. Der Grund dafür könnte in einer nicht abschließend geklärten Glukuronidierungsinteraktion liegen [33]. Auch hier gilt, dass durch standardmäßiges DrugMonitoring entsprechende Valproat-Dosisanpassungen ohne Schwierigkeiten erfolgen können. 


\section{Merke}

Die häufig eingesetzten Betalaktamantibiotika gelten als eher wenig anfällig für relevante unerwünschte Arzneimittelwechselwirkungen.

\section{Linezolid}

Linezolid gehört neben Tedizolid zur Substanzklasse der Oxazolidinone. Diese wirken bakteriostatisch über die selektive Hemmung der bakteriellen Proteinbiosynthese grampositiver Bakterien [34,35]. Linezolid wird stark durch p-Glykoprotein metabolisiert. Induktoren dieses Enzyms führen zu erniedrigten Linezolid-Spiegeln, Inhibitoren zu einer Spiegelerhöhung. Klinisch konnte dies besonders für Rifampicin (Induktor) und Clarithromycin (Inhibitor) gezeigt werden. Cytochrome spielen für die Linezolid-Biotransformationen dagegen keine Rolle [32].

\section{Merke}

Linezolid besitzt eine klinisch sehr ausgeprägte Monoaminoxidase hemmende Wirkung und kann daher insbesondere in Kombination mit Fentanyl oder Antidepressiva zu einem Serotoninsyndrom führen [20].

\section{Makrolide}

Makrolidantibiotika wirken bakteriostatisch über die selektive Hemmung der bakteriellen Proteinbiosynthese [36]. Sie werden selbst kaum durch andere intensivmedizinisch relevante Substanzen beeinflusst, können aber aufgrund ihrer potenten Hemmung von p-Glykoprotein und diversen Cytochromen zahlreiche andere Arzneimittel in deren Abbau hemmen. Dazu gehören insbesondere Esketamin, Statine, Protonenpumpenhemmer, Midazolam, Tacrolimus, Ciclosporin, Methylprednisolon sowie Oxycodon [32].

Erythromycin und Clarithromycin gelten darüber hinaus als Substanzen mit nachgewiesener QTc-Zeit-verlängernder Wirkung [12]. Daher empfiehlt es sich, bei gemeinsamer Anwendung mit anderen QTc-Zeit-beeinflussenden Medikamenten ( $\triangleright$ Tab.3) auf EKG-Veränderungen besonders zu achten.

\section{Fluorchinolone}

Fluorchinolone hemmen die bakterielle DNA-Gyrase (Topoisomerase II) sowie Topoisomerase IV, wodurch es zu DNA-Strangbrüchen und daraus folgender Zelllyse

\section{FALLBEISPIEL}

\section{Teil 2: Blutdruckentgleisung unter Antibiotikatherapie}

Die bei der Patientin nebenbefundlich seit mehreren Jahren bestehende schwere essenzielle Hypertonie war bereits mit einer antihypertensiven Sechsfachkombination aus Amlodipin, Bisoprolol, Clonidin, Chlortalidon, Doxazosin und Valsartan behandelt worden. Darunter erreichte die Patientin anamnestisch knapp normotensive Blutdruckwerte (140/70 mmHg bei Aufnahme). Im Verlauf des aktuellen Normalstationsaufenthalts hatten sich jedoch unter Beibehaltung der vorbestehenden Dosierungen die Blutdruckwerte deutlich erhöht. Selbst unter Hinzunahme weiterer Antihypertensiva wie Dihydralazin und Nitrendipin ließ sich keine Normotension mehr erreichen. Mit Blutdruckspitzen von 200/100 mmHg erfolgt daher die IMC-Verlegung.

Nach IMC-Aufnahme und eingehendem Aktenstudium wird als mutmaßliche Ursache für die Blutdruckentgleisung die antibiotische Therapie mit Rifampicin angesehen. Rifampicin ist ein ausgeprägter Induktor von p-Glykoprotein, diversen Cytochromen und UGT [8, 10,32]. Vier der 6 initial eingesetzten Antihypertensiva werden durch Rifampicin hinsichtlich einer beschleunigten Clearance negativ beeinflusst. Dies sind Amlodipin, Bisoprolol, Doxazosin und Valsartan [16], wodurch sich deren mangelhafter antihypertensiver Effekt während der Rifampicin-Komedikation erklären lässt. Die Tagesdosen der eingesetzten Substanzen sind in > Tab. 5 dargestellt.

- Tab. 5 Tagesdosen der eingesetzten Antihypertensiva.

\begin{tabular}{|c|c|c|c|c|c|c|c|c|c|c|c|}
\hline \multirow[t]{2}{*}{ Tag } & 1 & 2 & 3 & 4 & 5 & 6 & 7 & 8 & 9 & 10 & 11 \\
\hline & \multicolumn{6}{|c|}{ unter Rifampicin-Gabe } & \multicolumn{5}{|c|}{ Rifampicin beendet } \\
\hline Amlodipin $[\mathrm{mg}]^{*}$ & 10 & 10 & 10 & 10 & 10 & 10 & 10 & 10 & 10 & 10 & 10 \\
\hline Bisoprolol [mg] ${ }^{*}$ & 10 & 10 & 10 & 10 & 10 & 10 & 10 & 10 & 10 & 10 & 10 \\
\hline Clonidin $[\mu \mathrm{g}]$ & 475 & 475 & 475 & 475 & 475 & 475 & 475 & 475 & 475 & 475 & 475 \\
\hline Chlortalidon [mg] & 25 & 25 & 25 & 25 & 25 & 25 & 25 & 25 & 25 & 25 & 25 \\
\hline 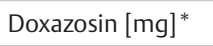 & 16 & 16 & 16 & 16 & 16 & 16 & 16 & 16 & 16 & 16 & 16 \\
\hline Valsartan [mg] ${ }^{*}$ & 320 & 320 & 320 & 320 & 320 & 320 & 320 & 320 & 320 & 320 & 320 \\
\hline Urapidil [mg] & 520 & 530 & 610 & 860 & 940 & 940 & 790 & 670 & 620 & 200 & 190 \\
\hline
\end{tabular}

* durch Rifampicin-Enzyminduktion beeinflusste Substanzen 
kommt [37]. Wie Makrolide werden auch Fluorchinolone durch andere Arzneimittel wenig beeinflusst. Cytochrome und p-Glykoprotein spielen beim Chinolonmetabolismus im Prinzip keine Rolle. Lediglich Ciprofloxacin ist ein starker CYP1A2-Inhibitor. Allerdings gibt es kaum intensivmedizinisch relevante CYP1A2-Substrate. Gelegentlich kommt Mirtazapin zur Koanalgesie oder schon als vorbestehendes Antidepressivum zum Einsatz, dessen Abbau gehemmt wird, und daher erhöhte Plasmaspiegel resultieren [10]. Ciprofloxacin, Levofloxacin und Moxifloxacin gelten als Substanzen mit nachgewiesener QTc-Zeitverlängernder Wirkung [12]. Wie auch schon bei den Makroliden empfiehlt es sich daher, bei gemeinsamer Anwendung mit weiteren Risikosubstanzen ( $\vee$ Tab.3) auf EKG-Veränderungen besonders zu achten.

\section{Rifampicin}

Rifampicin hemmt die bakterielle DNA-abhängige RNAPolymerase und daraus folgend die bakterielle Proteinbiosynthese mit überwiegend bakterizider Wirkung [38]. Es gilt in der Intensivmedizin als schwerwiegender Interaktionspartner. Grund dafür ist eine ausgeprägte Induktion von p-Glykoprotein, vieler relevanter Cytochrome sowie diverser UGT $[8,10,32]$. Wirkstoffspiegelverminderungen durch Rifampicin konnten für eine Vielzahl intensivmedizinisch relevanter Arzneistoffe in Studien nachgewiesen werden ( $\triangleright$ Tab. 1, Tab. 2).

\section{Merke}

Das Interaktionspotenzial von Rifampicin ist derart breit und komplex, dass sich bei dessen Anwendung im Intensivbereich grundsätzlich die Beratung durch einen klinischen Pharmazeuten empfiehlt.

\section{Antimykotika}

Azole

Azolantimykotika verursachen eine Hemmung der Ergosterolbiosynthese und somit eine Schädigung der mykotischen Zellmembran mit überwiegend fungistatischer Wirkung [39]. Azole wie Fluconazol und Voriconazol werden über CYP3A4, CYP2C9 sowie CYP2C19 metabolisiert. Gleichzeitig sind sie auch starke Inhibitoren dieser Enzyme. Daraus resultieren potenziell erhöhte Plasmaspiegel von Arzneistoffen, die über diese Cytochrome biotransformiert werden, bei gleichzeitiger Anwendung mit Azolantimykotika ( $\triangleright$ Tab. 2) $[8,10]$. Umgekehrt können starke Induktoren von CYP3A4, CYP2C9 und CYP2C19 die Plasmaspiegel von Azolen reduzieren. Am wenigsten durch solche Effekte beeinflusst wird Fluconazol, da es zu etwa $90 \%$ unverändert renal eliminiert wird. Der hepatische Metabolismus über Cytochrome spielt demnach für Fluconazol nur eine untergeordnete Rolle, weshalb diese Substanz aus pharmakokinetischer Sicht relativ interaktionsresistent ist [40]. Hingegen können stark verminderte Voriconazol-Plasmaspiegel bei kombinierter Anwendung mit Rifampicin, Phenytoin oder Carbamazepin auftreten [10].
Fluconazol, Voriconazol und Posaconazol können die QTc-Zeit verlängern [12]. Daher empfiehlt es sich, bei gemeinsamer Anwendung mit anderen QTc-Zeit-beeinflussenden Medikamenten ( $\triangleright$ Tab.3) auf EKG-Veränderungen besonders zu achten.

\section{Merke}

Azolantimykotika sind starke Cytochrominhibitoren mit breitem Interaktionsspektrum.

\section{Echinocandine}

Echinocandine hemmen die mykotische $\beta$-(1,3)-D-Glucansynthase, was zu einer erhöhten Zellmembranpermeabilität mit daraus resultierender Zelllyse führt [41]. Die klinisch verfügbaren Echinocandine zeigen substanzspezifische Unterschiede in ihrem Metabolismus.

So wird Caspofungin unabhängig vom Cytochom-P450System biotransformiert. Allerdings hemmt es p-Glykoprotein schwach und BRCP stark [42]. Im intensivmedizinischen Alltag bereitet dies in der Regel keine relevanten Probleme. Allerdings führt Ciclosporin zu erhöhten und Tacrolimus zu erniedrigten Caspofungin-Plasmaspiegeln bei gleichzeitiger Gabe. Die Ursache dieser Wechselwirkungen ist unklar [40]. Weiterhin wird Caspofungin durch Rifampicin, Dexamethason, Phenytoin und Carbamazepin in seinem Abbau beschleunigt. Auch die Ursache dieser Interaktion ist letztlich nicht eindeutig geklärt [43].

Anidulafungin wird wie Caspofungin CYP-unabhängig metabolisiert. Der Abbau erfolgt über eine spontane Substanzdegradation. Eine weitere Hydrolyse und N-Acetylierung geschehen unabhängig von Phase-II-Enzymen [40]. Anidulafungin ist ein starker Hemmstoff von BRCP [42]. Wie auch bei Caspofungin erhöht Ciclosporin die Plasmaspiegel von Anidulafungin. Tacrolimus zeigt diesbezüglich jedoch keine Interaktion [40].

Micafungin wird über CYP3A4 metabolisiert und hemmt dieses auch leicht [44]. Aufgrund dieses Effekts kann Micafungin die Plasmaspiegel von Ciclosporin erhöhen, ohne selbst von diesem beeinflusst zu werden [40]. Erstaunlicherweise zeigen selbst starke CYP3A4-Induktoren wie Rifampicin keinen wesentlichen Einfluss auf die Micafungin-Pharmakokinetik [45].

\section{Antiepileptika}

In der Neurointensivmedizin kommen Antiepileptika regelmäßig zur Anwendung. Dies sind insbesondere Valproat, Levetiracetam, Lacosamid, gelegentlich noch Phenytoin und in zunehmendem Maß auch Brivaracetam [46].

Valproat wird über CYP2C9, CYP2C19, CYP3A4 und UGT metabolisiert und hemmt diese auch schwach $[23,47]$. Starke CYP-Induktoren wie Rifampicin, Phenytoin und Carbamazepin führen zu einem beschleunigten Abbau 
von Valproat mit entsprechend erniedrigten Wirkstoffspiegeln. Valproat kann Diazepam aus dessen Plasmaproteinbindung verdrängen und somit zu einer erhöhten Fraktion von ungebundenem Diazepam führen mit entsprechender Wirkungsverstärkung. Weiterhin reduziert die Konkurrenz um UGT die Plasma-Clearance von Lorazepam um bis zu $40 \%$, wenn Valproat gleichzeitig angewendet wird [48]. Die Erhöhung der Plasmaspiegel von Propofol sowie der beschleunigte Valproat-Abbau durch Carbapeneme wurden bereits erläutert. Zusätzlich existieren Daten, die eine Hemmung der Serotoninwiederaufnahme durch Valproat zeigen, sodass prinzipiell ein Serotoninsyndrom ausgelöst werden kann, insbesondere in Kombination mit weiteren serotonergen Substanzen $(\triangleright$ Tab. 4) [13].

Im Gegensatz zu Valproat wird Levetiracetam durch Hydrolyse unabhängig von Cytochromen und Phase-II-Reaktionen metabolisiert [23]. Levetiracetam hemmt die Clearance von Methotrexat und erhöht daher dessen Plasmaspiegel [49]. Darüber hinaus kann Levetiracetam jedoch als wenig interaktionsrelevant eingestuft werden.

Lacosamid wird zu etwa 95\% renal eliminiert, 30\% davon als inaktiver Metabolit. Dieser wird über CYP2C19 gebildet. Bislang konnten für Lacosamid keine klinisch relevanten Interaktionen mit intensivmedizinisch gängigen Arzneimitteln nachgewiesen werden [46].

Brivaracetam als jüngste antiepileptische Substanz ist strukturell mit Levetiracetam verwandt, welches wie dieses primär enzymunabhängig hydrolysiert, dann jedoch über CYP2C19 weiter hydroxyliert wird. Insbesondere starke CYP-Induktoren wie Rifampicin, Phenytoin und Carbamazepin führen zu relevant erniedrigten Plasmaspiegeln von Brivaracetam [46].

Phenytoin gilt im Intensivbereich als schwerwiegender Interaktionspartner. Grund dafür ist eine ausgeprägte Induktion von p-Glykoprotein, vieler relevanter Cytochrome sowie diverser UGT und Epoxidhydrolasen [47]. Wirkstoffspiegelverminderungen durch Phenytoin konnten für eine Vielzahl intensivmedizinisch relevanter Arzneistoffe in Studien gesichert werden ( $\triangleright$ Tab. 1, Tab. 2).

\footnotetext{
Merke

Das Interaktionspotenzial von Phenytoin ist derart breit und komplex, dass sich bei dessen Anwendung im Intensivbereich grundsätzlich die Beratung durch einen klinischen Pharmazeuten empfiehlt.
}

Letztlich sollte bei Anwendung antiepileptischer Substanzen auf der Intensivstation grundsätzlich auch der kombinierte Einsatz von Arzneimitteln beachtet werden, die die Krampfschwelle senken können. Dies sind im Intensivbereich insbesondere Penicilline, Carbapeneme, Ery- thromycin, Levofloxacin, Linezolid, Haloperidol, SSRI und Trizyklika [23].

\section{Immunsuppressiva}

Wechselwirkungen bei Immunsuppressiva sind intensiv für die Calcineurininhibitoren Ciclosporin und Tacrolimus untersucht worden. Beide Substanzen werden sowohl über p-Glykoprotein als auch über CYP3A4 metabolisiert. Daher sind potenzielle Interaktionen mit Arzneimitteln zu erwarten, die diese Enzyme hemmen oder induzieren ( $\vee$ Tab. 1, Tab. 2). Klinisch relevant sind v. a. die Makrolide Erythromycin und Clarithromycin durch kombinierte p-Glykoprotein- und CYP3A4-Hemmung sowie die Azole Fluconazol und Voriconazol, allerdings durch alleinige CYP3A4-Inhibition. Unter diesen Kombinationen sind deutlich erhöhte Plasmaspiegel von Ciclosporin und Tacrolimus nachgewiesen worden. Starke Induktoren von p-Glykoprotein und CYP3A4 wie Rifampicin, Carbamazepin und Phenytoin senken die Ciclosporin- und Tacrolimus-Plasmaspiegel deutlich ab, sodass Rejektionen beschrieben sind. Diese treten sogar bei engmaschigem Einsatz eines therapeutischen Drug Monitorings auf [10]. Die Effekte der Calcineurininhibitoren auf Echinocandine wurden bereits erläutert.

Methylprednisolon und Hydrocortison sind ebenfalls Substrate von CYP3A4. Klinische Daten zeigen, dass wie auch bei den Calcineurininhibitoren v.a. Makrolide und Azolantimykotika zu klinisch relevanten Interaktionen führen, im Sinne einer verminderten Kortikoid-Clearance. Weiterhin reduziert insbesondere Rifampicin die zu erwartenden Plasmaspiegel von Methylprednisolon und Hydrocortison [50].

Der selektive und reversible Inhibitor der Inosinmonophosphatdehydrogenase Mycophenolat wird über UGT biotransformiert, mit einem zusätzlich klinisch nicht relevanten Anteil über CYP3A4. Es konnte in Pharmakokinetik-Studien ein signifikant beschleunigter MycophenolatMetabolismus bei Komedikation mit Rifampicin nachgewiesen werden [51]. Mycophenolat und dessen Glukuronide konkurrieren bei der tubulären Sekretion mit Aciclovir und Ganciclovir, deren Plasmaspiegel daher geringfügig erhöht werden [52]. Weiterhin unterliegt Mycophenolat einem ausgeprägten enterohepatischen Kreislauf. Sein Glukuronid wird im Kolon durch dort physiologisch vorkommende, überwiegend anaerobe Bakterien mit hoher $\beta$-Glukuronidase-Aktivität deglukuronidiert und so erneut resorbiert. Antibiotika mit überwiegender Aktivität gegen diese Standortflora können daher den enterohepatischen Kreislauf stören und zu erniedrigten Mycophenolat-Spiegeln führen. Dies sind insbesondere Aminoglykoside, Cephalosporine, Fluorchinolone und Penicilline [53]. 
Merke

Antibiotika mit Aktivität gegen Bakterien mit hoher $\beta$-Glucuronidase-Aktivität können den enterohepatischen Kreislauf von Mycophenolat stören.

\section{Prokinetika}

Die auf Intensivstationen gängigen Prokinetika sind Metoclopramid, Domperidon und Erythromycin [54]. Metoclopramid ist ein Serotonin-(5-HT4-)Rezeptor-Agonist sowie ein Dopamin-( $\left(D_{2}-\right)$ Rezeptor-Antagonist. Domperidon hemmt lediglich $\mathrm{D}_{2}$-Rezeptoren. Erythromycin aktiviert Motilin-Rezeptoren. Alle 3 Mechanismen führen zu einer Erhöhung der gastrointestinalen Motilität [55].

Metoclopramid wird über CYP2D6 metabolisiert. Starke Inhibitoren dieses Enzyms wie Amiodaron erhöhen daher die Plasmaspiegel von Metoclopramid. Bei gleichzeitiger Verabreichung mit anderen Dopaminantagonisten wie Haloperidol (z. B. im Rahmen einer Delirtherapie) ist das erhöhte Risiko für extrapyramidal-motorische Störungen zu beachten [56]. Weiterhin kann durch das serotonerge Potenzial von Metoclopramid v.a. in Kombination mit weiteren Risikosubstanzen in seltenen Fällen ein Serotoninsyndrom ausgelöst werden ( $\bullet$ Tab. 4) [13]

Domperidon wird hauptsächlich über CYP3A4 biotransformiert. Daher sind insbesondere durch starke Induktoren und Inhibitoren dieses Enzyms relevante Wechselwirkungen zu erwarten ( $\triangleright$ Tab. 2). Domperidon gehört zu den Substanzen mit sehr großem QTc-Zeit-verlängerndem Potenzial. Daher ist bei gleichzeitiger Verabreichung weiterer Arzneimittel mit Wirkung auf die QTc-Zeit Vorsicht geboten ( $\vee$ Tab. 4$)$. Dies gilt insbesondere für Substanzen, die zusätzlich auch eine CYP3A4-Inhibition zeigen, da durch sie eine Erhöhung der Domperidon-Plasmaspiegel zu erwarten ist [57].

Erythromycin unterliegt wie Domperidon einem CYP3A4-Metabolismus. Im Gegensatz zu diesem hemmt es selbst jedoch CYP3A4 in relevantem Ausmaß. Daher spielt Erythromycin als Interaktionspartner in der Intensivmedizin eine bedeutende Rolle ( $\bullet$ Tab. 2). Zahlreiche Wechselwirkungen wurden diesbezüglich bereits erläutert. Wie schon im Fall von Domperidon muss auch bei Erythromycin auf dessen hohes QTc-Zeit-verlängerndes Potenzial hingewiesen werden, mit den entsprechenden, bereits beschriebenen klinischen Konsequenzen. Interessant ist, dass Azithromycin offenbar eine vergleichbare prokinetische Wirkung zeigt, ohne jedoch über CYP3A4 metabolisiert zu werden, und daher ein deutlich günstigeres Wechselwirkungsprofil besitzt [54].

\section{Magensäure regulierende Therapeutika}

\section{Protonenpumpeninhibitoren}

Omeprazol, Esomeprazol und Pantoprazol werden in der Intensivmedizin zur Stressulkusprophylaxe und Therapie von Ulzera eingesetzt. Sie gehören mit den übrigen Protonenpumpenhemmern zu den Substanzen mit der stärksten Magensäuresuppression durch irreversible Hemmung der $\mathrm{H}^{+} / \mathrm{K}^{+}$-ATPase in den Belegzellen [26].

Omeprazol und Esomeprazol werden über Cytochrome metabolisiert und hemmen dabei CYP2C19 stark sowie CYP3A4 moderat. Insbesondere die Inhibition von CYP2C19 kann die Biotransformation des Prodrugs Clopidogrel zu dessen aktivem Metaboliten vermindern und somit die Thrombozytenaggregationshemmung negativ beeinflussen. Pantoprazol zeigt diese Wechselwirkung aufgrund fehlender CYP2C19-Affinität nicht [58].

Weiterhin erhöhen Omeprazol und Esomeprazol die Plasmaspiegel von Phenytoin und Citalopram durch CYP2C19-Hemmung. Darüber hinaus existieren widersprüchliche Daten bezüglich einer reduzierten Ciclosporin-Clearance bei Herz-, nicht jedoch bei Nierentransplantierten durch Omeprazol. In diesen klinischen Konstellationen empfiehlt sich dringend ein therapeutisches Drug-Monitoring von Ciclosporin [26]. Starke CYP3A4-Inhibitoren wie Fluconazol und Clarithromycin können ihrerseits die Wirkspiegel von Omeprazol und Esomeprazol erhöhen ( $\triangleright$ Tab. 2). Dies erfolgt jedoch in relevantem Ausmaß nur bei Patienten mit CYP2C19-Defizienz, da bei diesen der Hauptmetabolisierungsweg von CYP2C19 auf CYP3A4 ausweicht [59].

Merke

Pantoprazol erscheint hinsichtlich der Pharmakokinetik der am wenigsten anfällige Protonenpumpenhemmer zu sein.

\section{Histamin-( $\left.\mathrm{H}_{2}-\right)$ Antagonisten}

Ranitidin wird als parenteral verfügbarer $\mathrm{H}_{2}$-Antagonist auf Intensivstationen zur Stressulkusprophylaxe eingesetzt. Es wird nicht über das Cytochrom-P450-System metabolisiert. Sein klinisches Interaktionspotenzial ist daher vernachlässigbar [60]. 


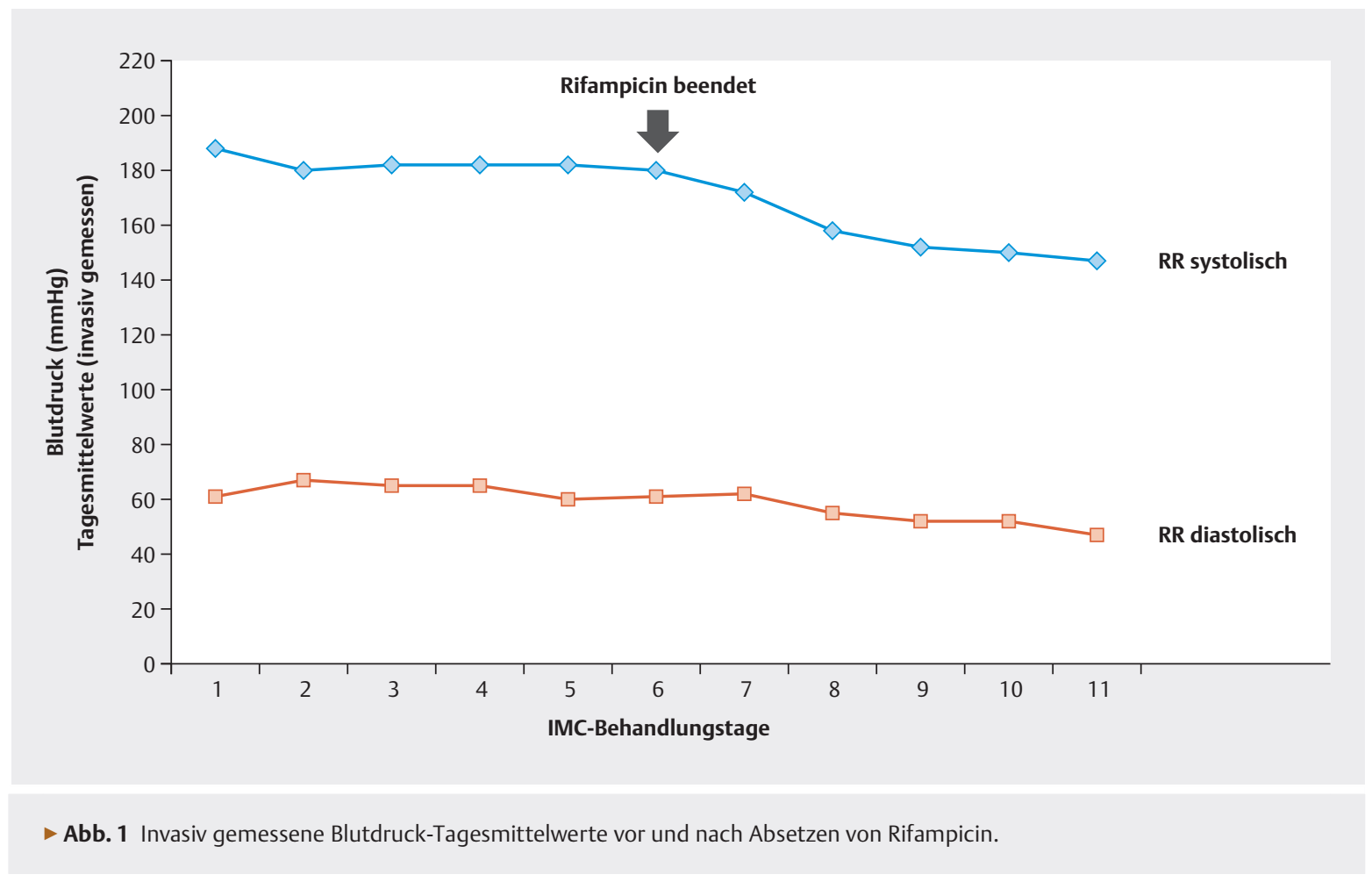

\section{Fazit}

Auf Intensivstationen ist aufgrund der regelmäßig notwendigen Polypharmakotherapie grundsätzlich mit potenziellen Arzneimittelinteraktionen zu rechnen. Die Anzahl der tatsächlich auftretenden klinisch relevanten Wechselwirkungen ist dabei jedoch schwer vorhersagbar. Profunde Kenntnisse von Pharmakokinetik und -dynamik der zumindest häufig eingesetzten Arzneimittel sind daher für die Therapiesicherheit von großer Bedeutung. Interaktionsdatenbanken können zusätzlich helfen, potenzielle Probleme aufzudecken. Am wichtigsten erscheint jedoch eine gute und regelmäßige Zusammenarbeit mit einem klinischen Pharmazeuten, der regelmäßig an intensivmedizinischen Visiten teilnimmt und die Patienten sowie deren Krankheitsverläufe kennt.

\section{FALLBEISPIEL}

Teil 3: Behandlung der Arzneimittelinteraktion und weiterer Verlauf

Nach Rücksprache mit den gefäßchirurgischen Kollegen soll die kalkulierte Rifampicin-Medikation vorerst beibehalten werden, da noch kein mikrobiologischer Erregernachweis aus den Gewebeproben der Revisionsoperation vorliegt. Daher wird zunächst zur weiteren antihypertensiven Therapie zusätzlich Urapidil verabreicht, da dieses durch Rifampicin pharmakokinetisch nicht beeinflusst wird [16]. Allerdings zeigt sich trotz hoher Tagesdosen kein zufriedenstellender Erfolg. Die Dosierungen der übrigen Antihypertensiva werden beibehalten ( $\vee$ Tab. 5 ).

Schließlich gelingt aus den intraoperativ gewonnenen Proben der mikrobiologische Nachweis von Proteus mirabilis und Morganella morganii, zweier gramnegativer Keime, die prinzipiell keiner Vancomycin-/Rifampicin-Therapie bedürfen, sodass eine alleinige Medikation mit Meropenem möglich ist. Nach Absetzen von Rifampicin normalisiert sich der Blutdruck zügig ( $\bullet$ Abb. 1). Das Urapidil kann in diesem Zug deutlich reduziert werden ( $\triangleright$ Tab. 5). Nach Rückverlegung auf die Normalstation konnten letztlich auch Meropenem und Urapidil beendet werden, und die Patientin wurde mit einem Blutdruck von 140/70 mmHg nach Hause entlassen. 


\section{KERNAUSSAGEN}

- Die Wahrscheinlichkeit des Auftretens von Arzneimittelwechselwirkungen hängt von der Anzahl der verordneten Arzneimittel ab.

- Es muss prinzipiell zwischen potenziellen und manifesten Arzneimittelinteraktionen unterschieden werden. Die klinisch tatsächlich auftretenden Wechselwirkungen sind deutlich seltener als die potenziell möglichen.

- Pharmakokinetische Interaktionen spielen in der Intensivmedizin die größte Rolle und sind systematisch gut einteilbar.

- CYP3A4 ist das Cytochrom mit dem breitesten Biotransformationsspektrum und stellt neben dem p-Glykoprotein die wichtigste Ursache für pharmakokinetische Arzneimittelwechselwirkungen dar.

- Wechselwirkungen mit Einfluss auf die Plasmaspiegel von Opioiden und Sedativa können durch standardmäßiges Monitoring von Analgesie und Sedierung auf Intensivstationen schnell erkannt werden.

- Die häufig eingesetzten Betalaktamantibiotika gelten als eher wenig anfällig für relevante unerwünschte Arzneimittelwechselwirkungen.

- Azolantimykotika sind starke Cytochrominhibitoren mit breitem Interaktionsspektrum.

- Das Interaktionspotenzial von Phenytoin und Rifampicin gilt in der Intensivmedizin als schwerwiegend.
Interessenkonflikt

Erklärung zu finanziellen Interessen

Forschungsförderung erhalten: nein; Honorar/geldwerten Vorteil für Referententätigkeit erhalten: nein; Bezahlter Berater/interner Schulungsreferent/Gehaltsempfänger: nein; Patent/Geschäftsanteile/Aktien (Autor/Partner, Ehepartner, Kinder) an im Bereich der Medizin aktiven Firma: nein; Patent/ Geschäftsanteile/Aktien (Autor/Partner, Ehepartner, Kinder) an zu Sponsoren dieser Fortbildung bzw. durch die Fortbildung in ihren Geschäftsinteressen berührten Firma: nein.

Erklärung zu nichtfinanziellen Interessen

Die Autorinnen/Autoren geben an, dass kein Interessenkonflikt besteht.

\section{Autorinnen/Autoren}

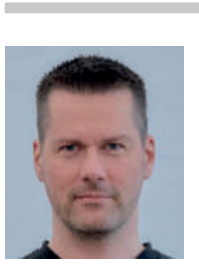

\section{Andreas Drust}

Dr. med. Andreas Drust studierte Pharmazie in Braunschweig sowie Medizin in Magdeburg und arbeitet als Oberarzt im Intensivbereich der Klinik für Anästhesiologie und Intensivtherapie des Universitätsklinikums Magdeburg. Er ist Apotheker sowie Facharzt für Anästhesiologie mit den Zusatzbezeichnungen Notfall- und Intensivmedizin.

\section{Korrespondenzadresse}

\section{Dr. med. Andreas Drust}

Klinik für Anästhesiologie und Intensivtherapie Universitätsklinikum Magdeburg

Leipziger Straße 44

39120 Magdeburg

andreas.drust@med.ovgu.de

Wissenschaftlich verantwortlich gemäß Zertifizierungsbestimmungen

Wissenschaftlich verantwortlich gemäß Zertifizierungsbestimmungen für diesen Beitrag ist Dr. med. Andreas Drust, Magdeburg.

\section{Zitierweise für diesen Artikel}

Anasthesiol Intensivmed Notfallmed Schmerzther 2020; 55; 300-313, doi:10.1055/a-0903-1767. 
[1] Böhm R, Reinecke K, Haen E et al. Arzneimittelinteraktionen Verstehen, vermitteln und vermeiden. Deutsche Apotheker Zeitung 2012; 36: 64

[2] Kane-Gill SL, Kirisci L, Verrico MM et al. Analysis of risk factors for adverse drug events in critically ill patients. Crit Care Med 2012; 40: 823-828

[3] Gallagher PF, Barry P], Ryan C et al. Inappropriate prescribing in an acutely ill population of elderly patients as determined by Beers' Criteria. Age Ageing 2008; 37: 96-101

[4] Fialová D, Topinková E, Gambassi G et al. Potentially inappropriate medication use among elderly home care patients in Europe. JAMA 2005; 293: 1348-1358

[5] Fitzmaurice MG, Wong A, Akerberg $\mathrm{H}$ et al. Evaluation of Potential Drug-Drug Interactions in Adults in the Intensive Care Unit: A Systematic Review and Meta-Analysis. Drug Saf 2019; 42: $1035-1044$

[6] Brunton LL, Chabner BA, Knollmann BC. Goodman \& Gilman's The Pharmacological Basis of Therapeutics. 12th ed. New York: Mcgraw-Hill Professional; 2011: 89-121

[7] Zagermann-Muncke P. Wenn Arzneistoffe Transportproteine beeinflussen. Pharmazeutische Zeitung 2006; 50. Im Internet: https://www.pharmazeutische-zeitung.de/ausgabe-502006/ abda-datenbank-wenn-arzneistoffe-transportproteinebeeinflussen/; Stand: 27.08.2019

[8] Kämmerer W. Klinisch relevante pharmakokinetische Arzneimittelinteraktionen in der Intensivmedizin. Med Klin Intensivmed Notmed 2011; 107: 128-140

[9] Liu YT, Hao HP, Liu CX et al. Drugs as CYP3A probes, inducers, and inhibitors. Drug Metab Rev 2007; 39: 699-721

[10] Spriet I, Meersseman W, de Hoon J et al. Mini-series: II. Clinical aspects. Clinically relevant CYP450-mediated drug interactions in the ICU. Intensive Care Med 2009; 35: 603-612

[11] Cascorbi I. Drug interactions - principles, examples and clinical consequences. Dtsch Arztebl Int 2012; 109: 546-556

[12] CredibleMeds. Im Internet: www.crediblemeds.org; Stand: 25.08.2019

[13] Wang RZ, Vashistha V, Kaur S et al. Serotonin syndrome: Preventing, recognizing, and treating it. Cleve Clin J Med 2016; 83: $810-817$

[14] Trescot AM, Datta S, Lee M et al. Opioid pharmacology. Pain Physician 2008; 11 (2. Suppl): 133-153

[15] Deutsche Gesellschaft für Anästhesiologie und Intensivmedizin (DGAI), Deutsche Interdisziplinäre Vereinigung für Intensiv- und Notfallmedizin (DIVI). S3-Leitlinie Analgesie, Sedierung und Delirmanagement in der Intensivmedizin (DAS-Leitlinie 2015). AWMF-Register Nr. 001/012. Im Internet: https:// www.awmf.org/leitlinien/detail/ll/001-012.html; Stand: August 2015

[16] Transformer - Metabolism of Xenobiotics Database. Im Internet: http://bioinformatics.charite.de/transformer/; Stand: 06.08.2019

[17] Armstrong SC, Cozza KL. Pharmacokinetic drug interactions of morphine, codeine, and their derivatives: theory and clinical reality, Part II. Psychosomatics 2003; 44: 515-520

[18] Armstrong SC, Cozza KL. Pharmacokinetic drug interactions of morphine, codeine, and their derivatives: theory and clinical reality, part I. Psychosomatics 2003; 44: 167-171

[19] Bundesinstitut für Arzneimittel und Medizinprodukte (BfArM). Rote-Hand-Briefe zu Fentany ${ }^{\circledR}$-janssen und Durogesic ${ }^{\circledR}$ SMAT $(12,25,50,75,100 \mu \mathrm{g} / \mathrm{h})$, transdermales Pflaster (Wirkstoff:
Fentanyl): Neuer Warnhinweis. Im Internet: www.bfarm.de/ SharedDocs/Risikoinformationen/Pharmakovigilanz/DE/RHB/ 2013/rhb-fentanyl-durogesic.html; Stand: 26.08.2019

[20] Linezolid: Komplexe Interaktion mit serotonergen Substanzen mit der Folge eines Serotonin-Syndroms. [Drug Safety Mail 2019-39] Arzneimittelkommission der deutschen Ärzteschaft: s.n., 10. Jul 2019

[21] Sahinovic MM, Struys MMRF, Absalom AR. Clinical Pharmacokinetics and Pharmacodynamics of Propofol. Clin Pharmacokinet 2018; 57: 1539-1558

[22] Dinis-Oliveira RJ. Metabolic Profiles of Propofol and Fospropofol: Clinical and Forensic Interpretative Aspects. Biomed Res Int 2018; 2018: 6852857. doi:10.1155/2018/6852857

[23] Farrokh S, Tahsili-Fahadan P, Ritzl EK et al. Antiepileptic drugs in critically ill patients. Crit Care 2018; 22: 153

[24] Rhabdomyolyse und mehr. Pharmazeutische Zeitung 2012; 56. Im Internet: www.pharmazeutische-zeitung.de/ausgabe052012/rhabdomyolyse-und-mehr/; Stand: 27.08.2019

[25] Olkkola KT, Ahonen J. Midazolam and other benzodiazepines. Handb Exp Pharmacol 2008; 182: 335-360

[26] Wedemeyer RS, Blume H. Pharmacokinetic drug interaction profiles of proton pump inhibitors: an update. Drug Saf 2014; 37: 201-211

[27] Uchaipichat V, Suthisisang C, Miners JO. The glucuronidation of $\mathrm{R}$ - and S-lorazepam: human liver microsomal kinetics, UDP-glucuronosyltransferase enzyme selectivity, and inhibition by drugs. Drug Metab Dispos 2013; 41: 1273-1284

[28] Tang JY, Kiang TKL, Ensom MHH. Pharmacokinetic Interactions between Valproic Acid and Lorazepam (PIVOtAL Study): A Review of Site-Specific Practices. Can J Hosp Pharm 2017; 70: $171-178$

[29] Fachinformation Dexdor 100 Mikrogramm/ml Konzentrat zur Herstellung einer Infusionslösung. s.l.: Orion Pharma, Januar 2019

[30] Keating GM. Dexmedetomidine: A Review of Its Use for Sedation in the Intensive Care Setting. Drugs 2015; 75: 1119-1130

[31] Bush K, Bradford PA. $\beta$-Lactams and $\beta$-Lactamase Inhibitors: An Overview. Cold Spring Harb Perspect Med 2016; 6: pii: a025247. doi:10.1101/cshperspect.a025247

[32] Bolhuis MS, Panday PN, Pranger AD et al. Pharmacokinetic Drug Interactions of Antimicrobial Drugs: A Systematic Review on Oxazolidinones, Rifamycines, Macrolides, FluoroquinoIones, and Beta-Lactams. Pharmaceutics 2011; 3: 865-913

[33] Mori H, Takahashi K, Mizutani T. Interaction between valproic acid and carbapenem antibiotics. Drug Metab Rev 2007; 39: 647-657

[34] ZYVOXID 2 mg/ml Infusionslösung. s.l.: Pfizer, Januar 2018

[35] Sivextro $200 \mathrm{mg}$ Pulver für ein Konzentrat zur Herstellung einer Infusionslösung. s.l.: MSD, August 2018

[36] Dinos GP. The macrolide antibiotic renaissance. Br J Pharmacol 2017; 174: 2967-2983

[37] Drlica K. Mechanism of fluoroquinolone action. Curr Opin Microbiol 1999; 2: 504-508

[38] Farr B, Mandell GL. Rifampin. Med Clin North Am 1982; 66: 157-168

[39] Schäfer-Korting M. New systemic antifungal drugs: mechanisms of action, drug interactions and side effects. Mycoses 2003; 46 (Suppl. 1): 28-31

[40] Bellmann R, Smuszkiewicz P. Pharmacokinetics of antifungal drugs: practical implications for optimized treatment of patients. Infection 2017; 45: 737-779 
[41] Patil A, Majumdar S. Echinocandins in antifungal pharmacotherapy. J Pharm Pharmacol 2017; 69: 1635-1660

[42] Lempers VJ, van den Heuvel J], Russel FG et al. Inhibitory Potential of Antifungal Drugs on ATP-Binding Cassette Transporters P-Glycoprotein, MRP1 to MRP5, BCRP, and BSEP. Antimicrob Agents Chemother 2016; 60: 3372-3379

[43] Johnson MD, Perfect JR. Caspofungin: first approved agent in a new class of antifungals. Expert Opin Pharmacother 2003; 4: 807-823

[44] Niwa T, Shiraga T, Takagi A. Effect of antifungal drugs on cytochrome P450 (CYP) 2C9, CYP2C19, and CYP3A4 activities in human liver microsomes. Biol Pharm Bull 2005; 28: 1805 1808

[45] Higashiyama $Y$, Kohno S. Micafungin: a therapeutic review. Expert Rev Anti Infect Ther 2004; 2: 345-355

[46] Orinx C, Legro B, Gaspard N. Recent antiseizure medications in the Intensive Care Unit. Minerva Anestesiol 2017; 83: 878887

[47] Zaccara G, Perucca E. Interactions between antiepileptic drugs, and between antiepileptic drugs and other drugs. Epileptic Disord 2014; 16: 409-431

[48] Fachinformation Valproat Chrono Winthrop. s.l.: SANOFI, Dezember 2018

[49] Fachinformation Keppra ${ }^{\circledR} 100 \mathrm{mg} / \mathrm{ml}$ Konzentrat zur Herstellung einer Infusionslösung. s.I.: UCB, März 2019

[50] Czock D, Keller F, Rasche FM et al. Pharmacokinetics and pharmacodynamics of systemically administered glucocorticoids. Clin Pharmacokinet 2005; 44: 61-98

[51] Staatz CE, Tett SE. Clinical pharmacokinetics and pharmacodynamics of mycophenolate in solid organ transplant recipients. Clin Pharmacokinet 2007; 46: 13-58
[52] Bullingham RE, Nicholls A], Kamm BR. Clinical pharmacokinetics of mycophenolate mofetil. Clin Pharmacokinet 1998; 34: $429-455$

[53] Fachinformation CellCept ${ }^{\circledR}$ i.v. s.l.: Roche, März 2018

[54] Youssef AS, Parkman HP, Nagar S. Drug-drug interactions in pharmacologic management of gastroparesis. Neurogastroenterol Motil 2015; 27: 1528-1541

[55] Camilleri M, Chedid V, Ford AC et al. Gastroparesis. Nat Rev Dis Primers 2018; 4: 41

[56] Fachinformation MCP-ratiopharm SF $10 \mathrm{mg} / 2 \mathrm{ml}$ Injektionslösung. s.l.: Ratiopharm, November 2018

[57] Fachinformation Motilium ${ }^{\circledR}$ Tropfen $10 \mathrm{mg} / \mathrm{ml}$ Suspension. s.l.: Takeda, November 2017

[58] Angiolillo DJ, Gibson CM, Cheng S et al. Differential effects of omeprazole and pantoprazole on the pharmacodynamics and pharmacokinetics of clopidogrel in healthy subjects: randomized, placebo-controlled, crossover comparison studies. Clin Pharmacol Ther 2011; 89: 65-74

[59] Blume H, Donath F, Warnke A et al. Pharmacokinetic drug interaction profiles of proton pump inhibitors. Drug Saf 2006; 29: 769-784

[60] Fachinformation Ranitidin-ratiopharm ${ }^{\circledR} 50 \mathrm{mg} / 5 \mathrm{ml}$ Injektionslösung. s.l.: Ratiopharm, Juli 2014

\section{Bibliografie}

DOI https://doi.org/10.1055/a-1153-2110

Intensivmedizin up2date 2020; 16: 163-176 @ Georg Thieme Verlag KG Stuttgart · New York ISSN 1614-4856 


\section{Punkte sammeln auf CMIE.thieme.de}

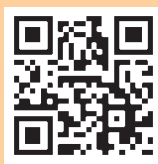

Diese Fortbildungseinheit ist in der Regel 12 Monate online für die Teilnahme verfügbar.

Den genauen Einsendeschluss finden Sie unter https://cme.thieme.de/.

Sollten Sie Fragen zur Online-Teilnahme haben, finden Sie unter https://cme.thieme.de/hilfe

eine ausführliche Anleitung. Wir wünschen viel Erfolg beim Beantworten

der Fragen!

Unter https://eref.thieme.de/CXEWFWT oder über den QR-Code kommen Sie direkt zur Startseite des Wissenstests.

VNR 2760512020158720611

\section{Frage 1}

Welche Aussage zur Interaktionspharmakokinetik trifft am wenigsten zu?

A In Phase I werden lipophile Substrate durch Oxidation, Reduktion oder Hydrolyse hydrophilisiert.

B Das Cytochrom mit dem breitesten Biotransformationsspektrum ist CYP3A4.

C CYP2D6 ist am häufigsten von Induktionseffekten betroffen.

D p-Glykoprotein wird vorrangig in Leber, Niere, Darm, Gehirn, Plazenta und Hoden exprimiert.

E Glukuronidierungsreaktionen gehören zur Phase II.

\section{Frage 2}

Welche Aussage zu Antiepileptika trifft am ehesten zu?

A Valproat hemmt Uridindiphosphatglukuronyltransferasen in relevantem Ausmaß.

B Phenytoin und Carbamazepin führen regelhaft zur Erhöhung der Plasmaspiegel von über CYP3A4 metabolisierten Arzneimitteln.

C Levetiracetam vermindert die Propofol-Clearance.

D Lacosamid ist mit dem Risiko eines Serotoninsyndroms assoziiert.

E Erythromycin, Levofloxacin, Linezolid sowie Haloperidol erhöhen die Krampfschwelle.

\section{Frage 3}

Welche Aussage zu Magensäure regulierenden Therapeutika trifft am ehesten zu?

A Pantoprazol ist ein starker CYP2C19-Inhibitor.

B Esomeprazol besitzt einen besonderen CYP2D6-Ausweichmetabolismus.

C Clopidogrel wird aufgrund seines Prodrug-Charakters nicht durch Protonenpumpeninhibitoren beeinflusst.

D CYP3A4-Inhibitoren wie Fluconazol und Clarithromycin können die Wirkspiegel von Omeprazol und Esomeprazol erhöhen.

E Ranitidin gilt in klinisch relevantem Ausmaß als interaktionsanfällig.

\section{Frage 4}

Welche Aussage zu Antibiotika trifft am wenigsten zu?

A Linezolid zeigt eine klinisch sehr ausgeprägte Monoaminoxidase hemmende Wirkung.

B Piperacillin kann die Clearance von Aminoglykosiden beschleunigen.

C Carbapenem-Plasmaspiegel werden durch Valproat deutlich erniedrigt.

D Erythromycin und Clarithromycin gelten als Substanzen mit nachgewiesener QTc-Zeit-verlängernder Wirkung.

E Ciprofloxacin ist ein starker CYP1A2-Inhibitor.

\section{Frage 5}

Welche Aussage zu Sedativa trifft am ehesten zu?

A Midazolam wird wie Propofol in relevantem Ausmaß über CYP2B6 metabolisiert.

B Dexmedetomidin ist ein günstiger Kombinationspartner für Betablocker, um Bradykardien zu vermeiden.

C Uridindiphosphatglukuronyltransferasen spielen beim Lorazepam-Metabolismus eine untergeordnete Rolle.

D Diazepam wird fast ausschließlich über CYP2C9 biotransformiert.

E Propofol kann in Komedikation mit weiteren Substanzen in seltenen Fällen eine Rhabdomyolyse auslösen.

\section{Frage 6}

Welche Aussage zu Immunsuppressiva trifft am ehesten zu?

A Ciclosporin und dessen Glukuronide konkurrieren bei der tubulären Sekretion mit Aciclovir und Ganciclovir.

B Antibiotika mit Aktivität gegen Bakterien mit hoher $\beta$-Glucuronidase-Aktivität können den enterohepatischen Kreislauf von Mycophenolat stören.

C Methylprednisolon und Hydrocortison werden durch CYP3A4-Inhibitoren nicht beeinflusst.

D Ciclosporin und Tacrolimus werden exklusiv über p-Glykoprotein metabolisiert.

E Calcineurininhibitoren erniedrigen die Plasmaspiegel aller Echinocandine.

- Weitere Fragen auf der folgenden Seite... 


\section{Punkte sammeln auf CME.thieme.de}

Fortsetzung $\ldots$

\section{Frage 7}

Welche Aussage zu Opioiden trifft am wenigsten zu?

A Fentanyl besitzt ein relevantes Risiko für das Auftreten eines malignen neuroleptischen Syndroms.

B Morphin und Hydromorphon sind Substrate von Uridindiphosphatglukuronyltransferasen.

C Amiodaron hemmt den Abbau von Buprenorphin.

D Opioid-Interaktionen führen auf Intensivstationen durch standardmäßiges Monitoring von Analgesie und Sedierung in der Regel zu wenig klinischen Problemen.

E Bei der gleichzeitigen Anwendung von Linezolid und Fentanyl ist Vorsicht geboten.

\section{Frage 8}

Welche Aussage zu Prokinetika trifft am ehesten zu?

A Erythromycin und Azithromycin unterscheiden sich in ihrem Interaktionspotenzial nur unwesentlich.

B Domperidon gehört zu den Substanzen mit sehr hohem QTcZeit-verlängerndem Potenzial.

C Metoclopramid gilt als wenig interaktionsanfällig.

D Dopaminantagonisten verhindern zuverlässig serotonerge Wechselwirkungen.

E Clarithromycin hemmt CYP1A2 in relevantem Ausmaß.

\section{Frage 9}

Welche Aussage zu Antimykotika trifft am ehesten zu?

A Starke CYP2D6-Induktoren reduzieren die Plasmaspiegel von Azolen.

B Posaconazol kann bei QTc-Zeitverlängerung problemlos eingesetzt werden.

C Caspofungin wird extensiv über das Cytochrom-P450-System biotransformiert.

D Micafungin reduziert die Plasmaspiegel von Ciclosporin.

E Aufgrund der hohen renalen Clearance spielen Interaktionen über Cytochrome für Fluconazol nur eine untergeordnete Rolle.

\section{Frage 10}

Welche Aussage zum im Beitrag geschilderten Fallbeispiel trifft am wenigsten zu?

A Plasmaspiegel von Amlodipin, Bisoprolol, Doxazosin und Valsartan werden durch Rifampicin negativ beeinflusst.

B Eine beeinträchtigte Wirkung der Antihypertensiva ist durch Meropenem nicht zu erwarten.

C Urapidil interagiert nicht in relevantem Ausmaß mit Rifampicin.

D Bei einem Verdacht auf Protheseninfektion mit ausschließlich gramnegativen Keimen ist eine Vancomycin-/RifampicinTherapie gerechtfertigt.

E Rifampicin induziert p-Glykoprotein, diverse Cytochrome sowie Uridindiphosphatglukuronyltransferasen. 